\title{
Genomic-Based Therapy of Gynecologic Malignancies
}

\author{
Maurie Markman ${ }^{1,2}$ \\ ${ }^{1}$ Cancer Treatment Centers of America, \\ Philadelphia, PA, ${ }^{2}$ Drexel University \\ College of Medicine, Philadelphia, PA \\ Correspondence: \\ maurie.markman@ctca-hope.com \\ Tel.: + 12155377502 \\ Fax.: + 12155377139
}

Received: 9 October 2018

Accepted: 15 March 2019

Key Words: Gynecologic Malignances - Ovarian Cancer - PARP Inhibitors • Microsatellite Instability - Endometrial Cancer.

\begin{abstract}
This paper will review the current status of genomic-based therapy of gynecologic malignancies. The routine "standard-of-care" delivery of targeted therapeutics based on the presence of specific molecular biomarkers in the management of the gynecologic malignancies has been delayed compared to the substantial progress made in several other tumor types. However, relatively recently reported and rather robust phase 3 trial data have confirmed a potentially major role for PARP inhibitors as both active treatment and maintenance therapy of advanced ovarian cancer. Further, data demonstrating the presence of a specific molecular phenotype (micro-satellite (instability high - MSI-H) is a valid biomarker for the potential clinical utility of checkpoint inhibitor immunotherapy has relevance for all gynecologic malignancies, and particularly in the setting of metastatic or recurrent endometrial cancer. Conclusions. The introduction of PARP inhibitors into the oncology armamentarium has substantially impacted standard-of-care strategies in the management of ovarian cancer. It is anticipated that the results of ongoing and future trials will further define the role of genomic-based therapy in ovarian cancer and other gynecologic malignancies.
\end{abstract}

\section{Introduction}

Advances in the chemotherapeutic management of ovarian cancer over the past several decades subsequently led to the introduction of several important anti-neoplastic drugs (cisplatin, carboplatin, paclitaxel) into the standard-of-care management of multiple malignancies (1). Unfortunately, the same cannot be stated for the role played by ovarian cancer, or any other gynecologic malignancy in the early development of molecularly-based ("genomic") therapeutic strategies. However, over just the past several years rapidly evolving paradigm-changing concepts of precision-cancer medicine have entered the arena of the gynecologic cancers and these changes are on the verge of transforming the fundamental management of ovarian cancer and other female pelvic malignancies.

The goal of this review will be to highlight recent advances in the delivery of targeted therapeutics in the management of the gynecologic malignancies.

\section{BRCA (BReast CAncer) Mutations and PARP (poly-[ADP-ribose] polymerase) Inhibitors}

Mutations within the Breast Cancer Susceptibility Gene (BRCA) 1 and 2 gene family have long been recognized to be responsible for the majority of ovarian cancers discovered to have a hereditary relationship 
(2). While data suggest such mutations are uncommon within the general population $(0.5 \%$ incidence in one large unselected patient study) as many as $10 \%-15 \%$ of women who develop ovarian cancer will be discovered to possess a germline BRCA abnormality $(2,3)$. An additional $5-7 \%$ of ovarian cancers will be found to have a somatic mutation in BRCA with a wildtype BRCA within the germline.

Of considerable interest to the question of possible therapeutic implications of the presence of a BRCA mutation investigators in several centers noted that the overall survival of patients treated with platinum-based chemotherapy and whose ovarian cancers contained this defect appeared to be somewhat superior to the much larger population of women with a wild-type (normal) BRCA gene $(4,5)$. (Note: Evidence also exists that the presence of a BRCA mutation may favorably impact the outcome associated with non-platinum-based chemotherapy $(6,7)$.

Subsequent pre-clinical investigative efforts revealed the major role of BRCA gene products in the DNA repair process and the impaired ability of malignant cells to adequately repair damage (including that produced by exposure to platinum agents) in the presence a mutation in the BRCA genes $(8,9)$. In experimental models poly (ADPribose) polymerase (PARP) was shown to be a second critical component of the DNA repair process. In fact, in a series of elegant studies investigators demonstrated that inhibition of PARP function in the presence of a genetic deficiency of BRCA produced a rather profound degree of tumor cell kill, while cells possessing a wild type BRCA were substantially less susceptible to the effects of PARP inhibition (1000-fold less sensitive) $(8,9)$.

This observation quickly led academic researches and biotech/pharma companies to initiate robust efforts to develop clinically useful inhibitors of PARP. Several agents in this class have been examined in trials in ovarian cancer and other malignances. Three PARP inhibitors are currently commercially available in the United States with regulatory approval granted for their administration in the management of ovarian cancer. Two PARP inhibitors (olaparib, rucaparib) are currently specifically approved for delivery as "therapy" of recurrent or persistent disease following several lines ( $\geq 2$ for rucapar$\mathrm{ib} ; \geq 3$ for olaparib) of cytotoxic chemotherapy in the presence of a BRCA mutation. Objective response rates have been reported to range between $30-70+\%$ in this setting with the greatest opportunity to achieve clinical benefit where there is also likely persistent sensitivity to platinum agents (10-17). It is important to acknowledge here that in the absence of formal randomized trial comparisons between the various PARP agents it is not possible to make any definitive statement regarding the relative clinical effectiveness of the individual drugs.

Three drugs (niraparib, olaparib, rucaparib) are approved as a "maintenance" approach following attainment of a clinical response (complete or partial) to a platinum-based second-line (or later) cytotoxic chemotherapy regimen (Table 1) (18-22). "Maintenance therapy" in this setting implies knowledge that the cancer remains present with the therapeutic goal to extend ("maintain") an achieved response with acceptable treatment-related side effects. The general concept is to continue therapy for an indefinite period, or until subsequent progression is documented, unacceptable toxicity develops, or a patient desires to discontinue treatment.

It is relevant to acknowledge here that the trials of PARP inhibitors in ovarian cancer have revealed the very impressive "sensitivity" and documented clinical benefit associated with tumors possessing either germline or somatic BRCA mutations (18, 23). However, even patients with a wild type 
Table 1. PARP Inhibitor Maintenance Therapy in Epithelial Ovarian Cancer

\begin{tabular}{llll}
\hline Drug (trial design) & $\begin{array}{l}\text { Patient } \\
\text { population }\end{array}$ & $\begin{array}{l}\text { Median PFS } \\
\text { (in months) }\end{array}$ & $\begin{array}{l}\text { Hazard } \\
\text { Ratio }^{+}\end{array}$ \\
\hline Niraparib (randomized phase 3) second-line (or later) (28) & Germline BRCA mutation & 21.0 vs. 5.5 & 0.27 \\
\cline { 2 - 4 } & Wildtype BRCA & 9.3 vs. 3.9 & 0.45 \\
\hline $\begin{array}{l}\text { Olaparib (randomized phase 2) second-line (or later) (19, } \\
\text { 20) }\end{array}$ & Overall population & 8.4 vs. 4.8 & 0.35 \\
\cline { 2 - 4 } & Germline BRCA mutation & 11.2 vs. 4.3 & 0.18 \\
\cline { 2 - 4 } & Wildtype BRCA & 7.4 vs 5.5 & 0.54 \\
\hline Olaparib (randomized phase 3) second-line (or later) (21) & Germline BRCA mutation & 19.1 vs 5.5 & 0.30 \\
\hline Olaparib (randomized phase 3) front-line (25) & Germline BRCA mutation & (Not reached) vs. 13.8 & 0.30 \\
\hline Rucaparib (randomized phase 3) second-line (or later) (22) & Germline BRCA mutation & 16.6 vs. 5.4 & 0.23 \\
\cline { 2 - 4 } & Intention to treat & 10.8 vs. 5.4 & 0.36 \\
\hline
\end{tabular}

PARP=Poly (ADP-ribose) polymerase; PFS=Progression-free survival; "Active Treatment vs. Placebo Control (all differences noted are "statistically significant"); ${ }^{\top}$ Treatment vs. Control; BRCA= Breast Cancer Susceptibility Gene.

BRCA (and no evidence of a somatic mutation) can respond to this class of drugs. The older term "BRCAness" had been employed to suggest the presence of additional poorly defined molecular abnormalities that interfered with DNA repair, in a manner like a BRCA mutation, and which might render cancer cells more susceptible to PARP inhibitors and platinum agents (24).

Researchers and molecular diagnostic companies are actively exploring possible algorism that may be employed in this clinical setting to identify such cancers that exhibit "homologous recombination deficiency" (HRD) independent of the presence of a documented BRCA mutation. However, while the presence of HRD with existing diagnostic platforms does appear to identify a population of individuals more likely to respond to a PARP inhibitor there remain a considerable percentage of patients whose cancers fail to exhibit this phenotype but who also achieve evidence of clinical benefit (based on the randomized phase 3 trial results compared to placebo). As a result, the FDA approval of the three available PARP drugs specifically did not require the presence of a BRCA mutation or of a HRD molecular phenotype to prescribe these agents.

Results of the first completed phase 3 randomized trial employing a PARP inhibi- tor (olaparib) as "maintenance" therapy for patients with a germline BRCA mutation in the front-line setting following the completion of a platinum and taxane regimen have recently been reported (25). Compared to "placebo" maintenance, treatment with olaparib resulted in a $70 \%$ reduction in the risk of disease progression or death. At three years follow-up $60 \%$ of patients treated with olaparib had not progressed compared to $27 \%$ who received placebo.

While the available oral PARP inhibitors differ somewhat in their toxicity profiles and schedule of administration they have all been shown to be reasonably well tolerated in the clinical trials setting, including the performance of formal quality-of-life assessments $(26,27)$. However, it is relevant to note that the administration of these agents results in a high incidence (approximately $70 \%)$ of so-called "low grade" nausea which in the context of daily oral therapy anticipated to be taken for several years may be far more serious to an individual patient's overall quality-of-life than the existing toxicity scale terminology might suggest. And the question to be asked here is of the willingness of a patient to continue to take an oral medication for this extended duration if she experiences "low grade" nausea every day. 


\section{Other Genomic Abnormalities of Therapeutic Relevance in the Gynecologic Malignancies}

Both high grade and low grade epithelial ovarian malignancies are characterized by a reasonably high incidence of molecular abnormalities including genes known or suspected to be "driver mutations" (e.g., PI3KCA, ADAMTS, DICER1, BRAF, KRAS, ARIDA1A; MEK; AKT2; PTEN, FBXW7) (28-34). However, either effective targeted therapeutics do not currently exist for these molecular events or the utility of agents revealed to be effective in other malignancies have not yet been shown to be relevant in ovarian cancer. Unfortunately, a similar conclusion can be drawn for the status of molecular targeting therapeutics in endometrial cancer (35).

A phase 2 study which examined the clinical activity in ovarian cancer of the established lung cancer anti-neoplastic, gefitinib (a tyrosine kinase inhibitor of EGFR) revealed only a single response ( $4 \%$ overall response rate) (36). However, this response occurred in the one patient in the trial whose cancer possessed an activating mutation in EGFR known to characterize the responding lung cancer patient population. The investigators also examined the incidence of such molecular events in ovarian cancer and revealed a rate of $3.5 \%$ ( 2 of 57 patients). Unfortunately, but certainly not surprisingly due to the low incidence of such mutations in ovarian cancer, this potentially highly relevant observation has not been followed-up with further essential clinical studies.

Similarly, an older phase 2 trial of trastuzumab in ovarian cancer discovered an overall incidence of overexpression of the Her2 receptor of only $11.4 \%$, a far lower proportion of patients than observed in breast cancer (37). In fact, a total of 837 patients were required to be screened to find the 45 patients who entered this phase 2 study. While the objective response rate was only
$7.3 \%$ it must be noted that the cancers of only 14 patients, $31 \%$ of study participants had +3 staining by immunohistochemistry for overexpression of Her2, the patient population with the highest probability of achieving a clinical response. The report did not describe the relationship between Her2 tissue staining and response, so it remains unknown today if the small subgroup of ovarian cancer patients $(<2 \% ; 14$ of 837 screened individuals) who strongly overexpress Her-2 within their cancers might be reasonable candidates to receive one or more of several known highly clinically active anti-Her2 therapeutic agents.

The documented presence of micro-satellite instability (MSI-H) is associated with the presence of multiple molecular abnormalities within a cancer cell and this event and has been shown represent a malignant phenotype with a reasonably high statistical probability (approximately $40 \%-50 \%$ ) of responding to an immune modulatory checkpoint inhibitor $(38,39)$. Based on data from several small, but impressive studies, the FDA has approved the commercial use of this strategy in the presence of the MSI-H phenotype, completely agnostic to the specific tumor type. (Note: This was the first time the FDA has approved the use of an anti-neoplastic agent completely independent of the site of tumor origin.

Overall, approximately $17 \%, 2 \%$ and $3.5 \%$ of endometrial, ovarian, and cervical cancers, respectively, will be found to be MSI-H if the tumors are subjected to specific molecular testing for the presence of this abnormality or the diagnostic platform utilized examines for multiple genomic events (40).

\section{Conclusions}

While to date genomic abnormalities within two genes (BRCA 1 and 2) has dominated both research efforts and clinical use of precision medicine within the domain of the fe- 
male pelvic malignancies, it can be anticipated that this situation will substantially change over the coming years due to efforts by the pharmaceutical/biotech industry to develop novel products designed to effectively "target" both relatively common and unique molecular events within this group of cancers.

Conflict of Interest: The author declares that he has no conflict of interest.

\section{References}

1. Hennessy BT, Coleman RL, Markman M. Ovarian cancer. Lancet. 2009;374(9698):1371-82.

2. Neff RT, Senter L, Salani R. BRCA mutation in ovarian cancer: testing, implications and treatment considerations. Ther Adv Med Oncol. 2017;9(8):519-31.

3. Manickam K, Buchanan AH, Schwartz MLB, Hallquist MLG, Williams JL, Rahm AK, et al. Exome Sequencing-Based Screening for BRCA1/2 Expected Pathogenic Variants Among Adult Biobank Participants. JAMA Netw Open. 2018;1(5):e182140

4. Candido-dos-Reis F, Song H, Goode EL, Cunningham JM, Fridley BL, Larson MC, et al. Germline mutation in BRCA1 or BRCA2 and ten-year survival for women diagnosed with epithelial ovarian cancer. Clin Cancer Res. 2015;21(3):652-7.

5. Zhong Q, Peng H-L, Zhao X, Zhang L, Hwang W-T. Effects of BRCA1- and BRCA2-related mutations on ovarian and breast cancer survival: A meta-analysis. Clin Cancer Res. 2014;21(1):211-20.

6. Kaye SB, Lubinski J, Matulonis U, Ang JE, Gourley C, Karlan BY, et al. Phase II, open-label, randomized, multicenter study comparing the efficacy and safety of olaparib, a poly(ADP-ribose) polymerase inhibitor, and pegylated liposomal doxorubicin in patients with BRCA1 or BRCA2 mutations and recurrent ovarian cancer. J Clin Oncol. 2012;30(4):372-9.

7. Safra T, Rogowski O, Muggia FM. The effect of germ-line BRCA mutations on response to chemotherapy and outcome of recurrent ovarian cancer. Int J Gynecol Cancer. 2014;24(3):488-95.

8. Ashworth A. A synthetic lethal therapeutic approach: Poly(ADP) ribose polymerase inhibitors for the treatment of cancers deficient in DNA double-strand break repair. J Clin Oncol. 2008;26(22):3785-90.

9. Lord CJ, Ashworth A. PARP inhibitors: Synthetic lethality in the clinic. Science. 2017;355(6330): 1152-8.
10. Fong PC, Boss DS, Yap TA, Tutt A, Peijun W, Mergui-Roelvink $\mathrm{M}$, et al. Inhibition of poly (ADP-ribose) polymerase in tumors from BRCA mutation carriers. N Engl J Med. 2009;361(2):123-4.

11. Fong PC, Yap TA, Boss DS, Carden CP, MerguiRoelvink M, Gourley C, et al. Poly (ADP)-ribose polymerase inhibition: frequent durable responses in BRCA carrier ovarian cancer correlating with platinum-free interval. J Clin Oncol. 2010;28(15):2512-9.

12. Gelmon KA, Tischkowitz M, Mackay H, Swenerton K, Robidoux A, Tonkin K, et al. Olaparib in patients with recurrent high-grade serous or poorly differentiated ovarian carcinoma or triplenegative breast cancer: a phase 2, multicenter, open-label, non-randomized study. Lancet Oncol. 2011;12(9):852-61.

13. Audeh MW, Carmichael J, Penson RT, Friedlander M, Powell B, Bell-McGuinn KM, et al. Oral poly (ADP)-ribose polymerase inhibitor olaparib in patients with BRCA1 or BRCA2 mutations and recurrent ovarian cancer: A proof-of-concept trial. Lancet. 2010;376(9737):245-51.

14. Kaufman B, Shapria-Frommer R, Schmutzler RK, Audeh W, Friedlander M, Balmana J, et al. Olaparib monotherapy in patients with advanced cancer and a germline BRCA1/2 mutation. J Clin Oncol. 2015;33(3):244-50.

15. Domchek SM, Aghajanian C, Shapira-Frommer R, Schmutzler RK, Audeh MW, Friedlander M, et al. Efficacy and safety of olaparib monotherapy in germline BRCA1/2 mutation carriers with advanced ovarian cancer and three or more lines of prior therapy. Gynecol Oncol. 2016;140(2):199-203.

16. Swisher EM, Lin KK, Oza AM, Giordano $\mathrm{H}$, Konecny G, Coleman RL, et al. Rucaparib in relapsed, platinum-sensitive high-grade ovarian carcinoma (ARIEL 2 Part 1): an international, multicentre, open-label, phase 2 trial. Lancet Oncol. 2017;18(1):75-87.

17. Oza AM, Tinker AV, Oaknin A, Shapira-Frommer R, McNeish IA, Swisher EM, et al. Antitumor activity and safety of the PARP inhibitor rucaparib in patients with high-grade ovarian carcinoma and a germline or somatic BRCA1 or BRCA2 mutation: Integrated analysis of data from Study 10 and ARIEL2. Gynecol Oncol. 2017;147(2):267-75.

18. Mizra MR, Monk BJ, Herrstedt AM, Oza S, Mahner A, Redondo MF, et al. Niraparib maintenance therapy in platinum-sensitive, recurrent ovarian cancer. N Engl J Med. 2016;375(22):2154-64.

19. Ledermann J, Harter P, Gourley C, Friedlander M, Vergote I, Rustin G, et al. Olaparib maintenance therapy in platinum-sensitive relapsed ovarian cancer. N Engl J Med. 2012;366(15):1382-92. 
20. Ledermann J, Harter P, Gourley C, Friedlander M, Vergote I, Rustin G, et al. Olaparib maintenance therapy in patients with platinum-sensitive relapsed serious ovarian cancer: a preplanned retrospective analysis of outcomes by BRCA status in a randomized phase 2 trial. Lancet Oncol. 2014;15(8):852-61.

21. Pujade-Lauraine E, Lederemann JA, Selle F, Gebski V, Person RT, Oza AM, et al. Olaparib tablets as maintenance therapy in patients with platinumsensitive, relapsed ovarian cancer and a BRCA $1 / 2$ mutation (SOLO2/ENGOT-Ov21): a doubleblind, randomized, placebo-controlled, phase 3 trial. Lancet Oncol. 2017;18(9):1274-84.

22. Coleman RL, Oza AM, Lorusso D, Aghajanian C, Oaknin A, Dean A, et al. Rucaparib maintenance treatment for recurrent ovarian carcinoma after response to platinum therapy (ARIEL3): a randomized, double-blind, placebo-controlled, phase 3 trial. Lancet Oncol. 2017;390(10106):1949-61.

23. Hennessy BTJ, Timms KM, Carey MS, Gutin A, Meyer LA, Flake DD, et al. Somatic mutations in BRCA 1 and BRCA 2 could expand the number of patients that benefit from Poly(ADP ribose) polymerase inhibitors in ovarian cancer. J Clin Oncol. 2010;28(22):3570-6.

24. Bast RC, Mills GB. Personalizing therapy for ovarian cancer: BRCAness and beyond. J Clin Oncol. 2010;28(22):3545-8.

25. Moore K, Colombo N, Scambia G, Kim BG, Oaknin A, Friedlander M, et al. Maintenance olaparib in patients with newly diagnosed advanced ovarian cancer. N Engl J Med. 2018;379(26):2495-505.

26. Friedlander M, Gebski V, Gibbs E, Davies L, Bloomfield R, Hilpert F, et al. Health-related quality of life and patient-centred outcomes with olaparib maintenance after chemotherapy in patients with platinum-sensitive, relapse ovarian cancer and a BRCA1/2 mutation (SOLO/ENGOT Ov-21): a placebo-controlled, phase 3 randomized trial. Lancet Oncol. 2018;19(8):1126-34.

27. Oza AM, Mataulonis A, Malander S, Hidgens S, Sehouli J, del Campo JM, et al. Quality of life in patients with recurrent ovarian cancer treated with niraparib versus placebo (ENGOT-OV16/ NOVA): results from a double-blind, phase 3, randomized controlled trial. Lancet Oncol. 2018;19(8):1117-25.

28. Bregar AJ, Growdon WB. Emerging strategies for targeting PI3K in gynecologic cancer. Gynecol Oncol. 2016;140(2):333-44.

29. Liu Y, Yasukawa M, Chen K, Hu L, Broaddus RR, Ding L, et al. Association of somatic mutations of ADAMTS genes with chemotherapy sensitiv- ity and survival in high-grade ovarian carcinoma. JAMA Oncol. 2015;1(4):486-94.

30. Heravi-Moussavi A, Anglesio MS, Cheng SW, Senz J, Yang W, Prentice L, et al. Recurrent somatic DICER1 mutations in nonepithelial ovarian cancers. N Engl J Med 2012;366(3):234-42.

31. Kaldawy A, Segev Y, Lavie O, Auslender R, Sopik V, Narod SA. Low-grade serious ovarian cancer: A review. Gynecol Oncol. 2016;143(2):433-8.

32. Groen RS, Gershenson DM, Fader AN. Updates and emerging therapies for rate epithelial ovarian cancers: One size no longer fits all. Gynecol Oncol. 2015;136(2):373-83.

33. Miller CR, Oliver KE, Farley JH. MEK1/2 inhibitors in the treatment of gynecologic malignancies. Gynecol Oncol. 2014;133(1):128-37.

34. Elvin JA, Chura J, Gay LM, Markman M. Comprehensive genomic profiling of ovarian clear cell carcinomas identifies clinically relevant genomic alterations and targeted therapy options. Gynecol Oncol Rep. 2017;20:62-6.

35. Lherueux S, McCourt C, Rimel BJ, Duska L, Fleming G, Mackay H, et al. Moving forward with actionable therapeutic targets and opportunities in endometrial cancer: A NCI clinical trials planning meeting report. Gynecol Oncol. 2018 Feb 22. [Epub ahead of print].

36. Schilder RJ, Sill MW, Chen X, Darcy KM, Decesare SL, Lewandowski G, et al. Phase II study of gefitinib in patients with relapsed or persistent ovarian or primary peritoneal carcinoma and evaluation of epidermal growth factor receptor mutations and immunohistochemical expression: A Gynecologic Oncology Group Study. Clin Cancer Res. 2005;11(15):5539-48.

37. Bookman MA, Darcy KM, Clarke-Pearson D, Boothby RA, Horowitz IR. Evaluation of monoclonal humanized anti-Her2 antibody, trastuzumab, in patients with recurrent or refractory ovarian or primary peritoneal carcinoma with overexpression of Her2: A phase 2 trial of the Gynecologic Oncology Group. J Clin Oncol. 2003;21(2):283-90.

38. Le DT, Uram JN, Wang H, Bartlett BR, Kemberline $\mathrm{H}$, Eyring $\mathrm{AD}$, et al. PD-1 blockade in tumors with mismatch-repair deficiency. N Engl J Med. 2015;372(26):2509-20.

39. Ribas A, Wolchok JD. Cancer immunotherapy using checkpoint blockade. Science. 2018;359(6382): 1350-5

40. Le DT, Durham JN, Smith KN, Wang H, Bartlett BR, Aulakh LK, et. al. Mismatch repair deficiency predicts response of solid tumors to PD-1 blockade. Science. 2017;357(6349):409-13. 